\title{
Rapid Melt Growth of Single Crystal InGaAs on Si Substrates
}

\author{
Xue Bai, ${ }^{1}$ Chien-Yu Chen, ${ }^{2}$ Niloy Mukherjee, ${ }^{3}$ Peter B. Griffin, ${ }^{2}$ and James D. Plummer ${ }^{2}$ \\ ${ }^{1}$ Department of Materials Science and Engineering, Stanford University, Stanford, CA 94305, USA \\ ${ }^{2}$ Department of Electrical Engineering, Stanford University, Stanford, CA 94305, USA \\ ${ }^{3}$ Technology and Manufacturing Group, Intel Corporation, Hillsboro, OR 97124, USA
}

Correspondence should be addressed to Xue Bai; xuebai@stanford.edu

Received 29 June 2016; Revised 1 September 2016; Accepted 6 September 2016

Academic Editor: Pavel Lejcek

Copyright (C) 2016 Xue Bai et al. This is an open access article distributed under the Creative Commons Attribution License, which permits unrestricted use, distribution, and reproduction in any medium, provided the original work is properly cited.

InGaAs integration on Si substrates is an important topic for next generation electronic devices. Rapid melt growth (RMG) has the potential to grow defect-free lattice mismatched materials on Si at low cost. Most previous publications have focused on growing binary III-V compounds by RMG, but none have discussed ternary compound materials. In this paper, we demonstrate the RMG of the single crystal ternary compound InGaAs on Si substrates. We discuss two main issues. The first is segregation along the stripe length. An analytical model is developed to describe the segregation of In/Ga in the grown stripe and the model is compared with experimental data. The second issue is the dissolution of the Si seed region during RMG, which leads to formation of Si islands inside the InGaAs stripe. The results of this study are applicable to any compound material in which $\mathrm{Si}$ is soluble at the elevated temperatures required for RMG.

\section{Introduction}

As the scaling of Si devices has become more challenging, researchers have been searching for alternative channel materials to enhance Si capabilities [1]. InGaAs has attracted a lot of attention [2] due to its high mobility and low leakage current. Integrating InGaAs on Si [3] could allow novel functionalities like optoelectronics on low cost $\mathrm{Si}$ substrates. There have been several approaches to integrate InGaAs on $\mathrm{Si}$, but most of them are not easily applicable to large-scale manufacturing. For instance, direct epitaxial growth of large area InGaAs on $\mathrm{Si}$ [4] requires a thick buffer layer to attempt to accommodate lattice mismatches. This complicates integrating InGaAs devices with underlying $\mathrm{Si}$ devices. Wafer bonding uses expensive III-V substrates that are not compatible with large diameter Si wafers [5]. Local epitaxial growth in small seed holes [6,7] can help to confine defects due to lattice mismatch but this method has not yet been applied to large-scale manufacturing. RMG, on the other hand, can avoid many of the disadvantages of the above techniques by terminating the defects generated by lattice mismatch to a confined neck region near a seed window [8]. To date, many publications have successfully demonstrated high quality materials [9-11] grown on Si by RMG. However, no publications have described the RMG of any ternary compound materials such as InGaAs.

In this study, we conduct RMG experiments on amorphous InGaAs samples deposited on an insulator with a small seed window to the Si substrate and study their crystal quality by Electron Backscatter Diffraction (EBSD). The segregation behavior of $\mathrm{In} / \mathrm{Ga}$ atoms and Si dissolution from the seed in the RMG stripe were measured by Auger Electron Spectroscopy (AES). The Si dissolution from the seed is similar to that shown previously for Ge stripes [12] but is exacerbated by the low eutectic melting temperature between III-V materials and Si.

\section{Materials and Methods}

To prepare the sample substrates, we deposited approximately $50 \mathrm{~nm}$ thick silicon nitride films by Low Pressure Chemical Vapor Deposition (LPCVD) on clean Si (100) substrates. Positive photoresist was then coated, exposed, and developed to pattern $4 \mu \mathrm{m} \times 6 \mu \mathrm{m}$ seed windows. Plasma etching was then used to remove the top $40 \mathrm{~nm}$ silicon nitride film in seed windows, followed by hot phosphoric acid etch 


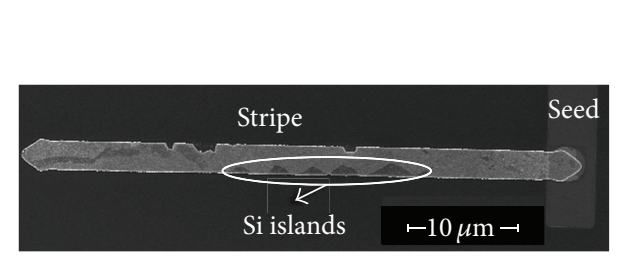

(a)

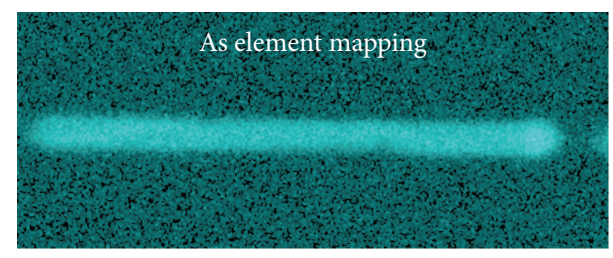

(c)

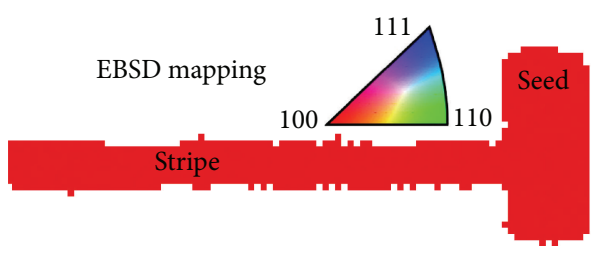

(b)

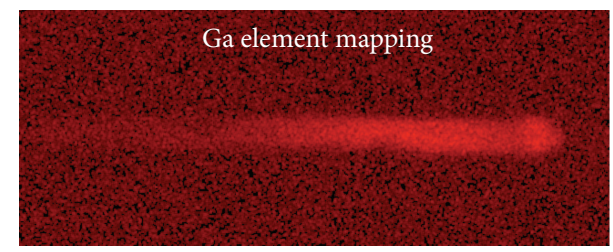

(d)



(e)

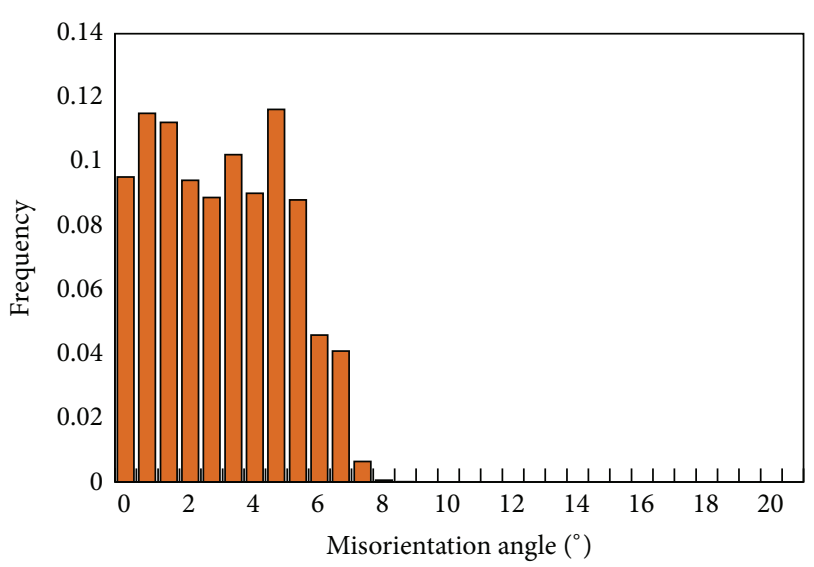

(f)

FIGURE 1: (a) SEM image of RMG InGaAs. Dark regions in the white circle are Si islands. (b) EBSD image of the crystal orientation. (c) EDS map for As element of the same InGaAs stripe. (d) EDS map for Ga element of the same InGaAs stripe. (e) EDS map for In element of the same InGaAs stripe. (f) Misorientation distribution of the InGaAs stripe.

to remove the remaining $10 \mathrm{~nm}$ of nitride to expose the underlying Si seed. We deposited $100 \mathrm{~nm}$ thick amorphous $\mathrm{In}_{0.27} \mathrm{Ga}_{0.2} \mathrm{As}_{0.53}$ films by molecular beam epitaxy (MBE) and the amorphous quality was verified by in situ reflection highenergy electron diffraction (RHEED) patterns. We patterned the InGaAs films into $2 \mu \mathrm{m} \times 10,25$, and $45 \mu \mathrm{m}$ stripes using the same positive photoresist process used for the seed windows followed by plasma etching the stripes with chlorine chemistries. The whole structure was then capped with a $1 \mu \mathrm{m}$ thick LPCVD $\mathrm{SiO}_{2}$ layer. Melt growth was performed in a standard rapid thermal process tool in a nitrogen ambient. The samples were heated at a rate of $20^{\circ} \mathrm{C} / \mathrm{s}$. Once the sample temperature reached the highest annealing temperature of $1145^{\circ} \mathrm{C}$, it was held for 1 second before cooling down. Cooling rates of $15^{\circ} \mathrm{C} / \mathrm{s}, 30^{\circ} \mathrm{C} / \mathrm{s}, 50^{\circ} \mathrm{C} / \mathrm{s}$, and $100^{\circ} \mathrm{C} / \mathrm{s}$ were used. AES was used to measure the composition of different elements near the sample surface. AES measures the concentration by detecting the kinetic energy and intensity of the Auger peaks. The resolution of the AES system was around $8 \mathrm{~nm}$. EBSD inverse pole figure maps were generated to visualize the crystal orientation of the sample. EBSD measures the orientation by identifying the Kikuchi bands from the diffracted electron beam from the sample surface. The resolution of EBSD is about $500 \mathrm{~nm}$.

\section{Results and Discussion}

The annealed InGaAs stripes were characterized by Electron Backscatter Diffraction (EBSD) [13] to check their crystal quality. Figure 1(a) shows the equivalent SEM picture of the measured stripe. The dark regions in the stripe are the $\mathrm{Si}$ islands. Figure $1(\mathrm{~b})$ is the EBSD map of an InGaAs stripe after RMG. The uniform red color of the stripe suggests that the InGaAs is (100) orientated single crystal.

Figures 1(c), 1(d), and 1(e) are the energy-dispersive Xray spectroscopy (EDS) maps of As, Ga, and In atoms. It can be seen that $\mathrm{Ga}$ is heavily concentrated at the front of the stripe near the seed region and In atoms segregate towards the end of the stripe. The nonuniform distribution of In and $\mathrm{Ga}$ atoms suggests a segregation effect similar to $\mathrm{SiGe} \mathrm{RMG}$ [12]. The misorientation distribution of the same InGaAs stripe is shown in Figure 1(f). It can be seen that most misorientation angles appeared between $0^{\circ}$ and $9^{\circ}$. The maximum misorientation angle is $9^{\circ}$. 


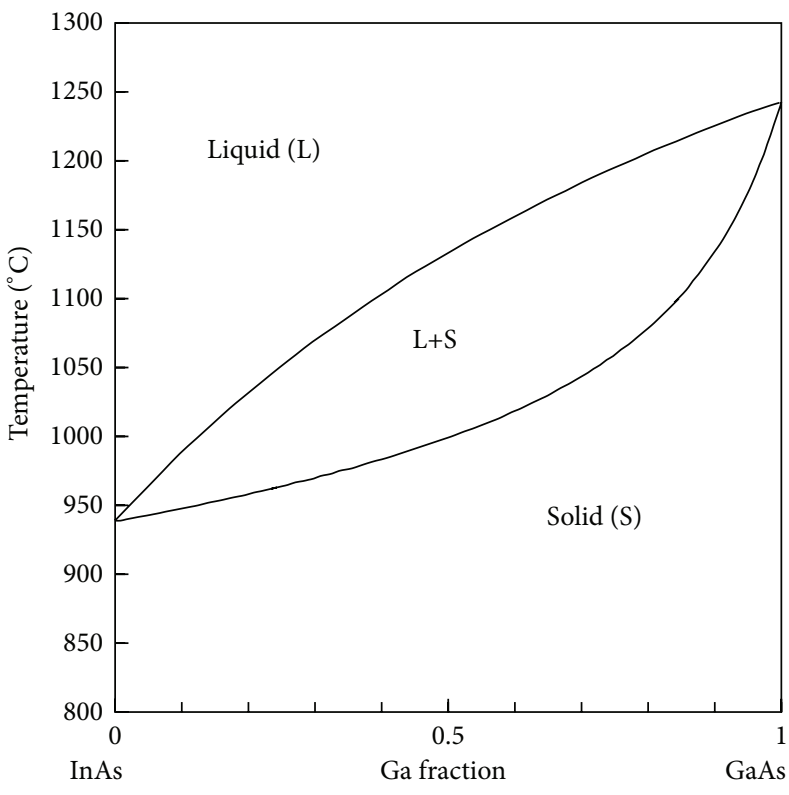

(a)

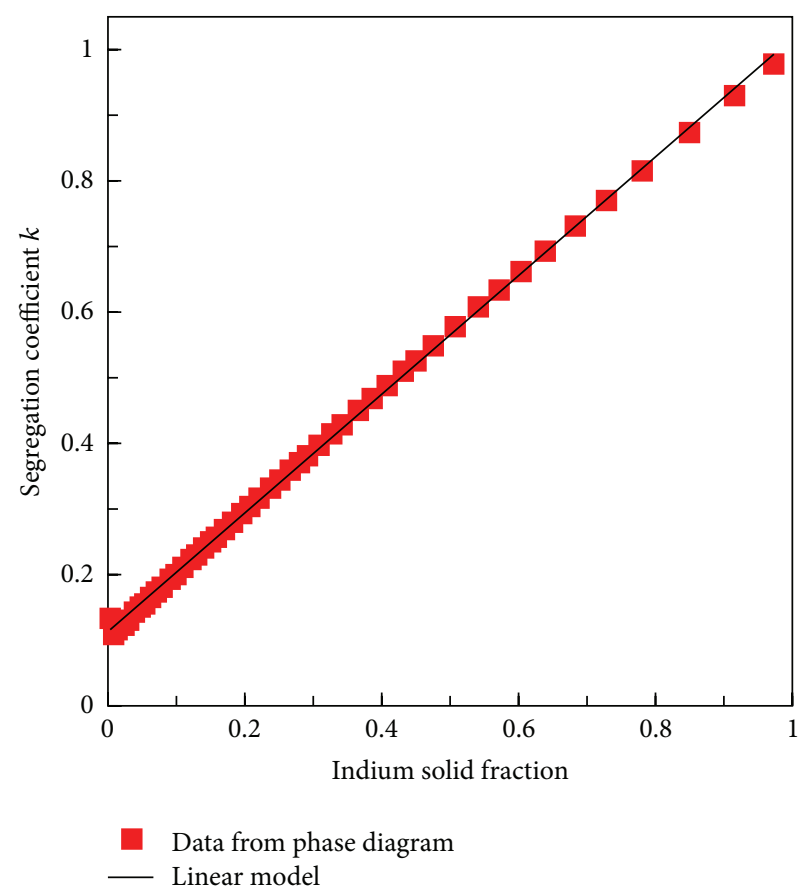

(b)

FIgURE 2: (a) Phase diagram of InGaAs. (b) Segregation coefficient is a linear function of indium solid concentration from phase diagram.

The segregation effects can be understood by considering the InGaAs phase diagram. InGaAs has a pseudobinary phase diagram [14], where InAs and GaAs can form a solid solution at any concentration. Figure 2(a) shows the lens-shaped phase diagram of InGaAs, which suggests that the modeling approach previously used for $\mathrm{Si} / \mathrm{Ge}$ segregation can also be applied to InGaAs. We develop an analytical solution similar to the SiGe RMG case [12] to calculate the segregation for any given initial concentration.

The differential form of the Scheil equation [15] is adopted here rather than the Scheil equation itself, since the segregation coefficient is not a constant. Equation (1) shows the differential form of the Scheil equation. $x_{L}$ represents the average liquid concentration in the melt and $x_{S}$ is the solid concentration at the liquid/solid interface during crystallization. $f_{S}$ is the fraction of the stripe.

$$
\left(x_{L}-x_{S}\right) d f_{S}=\left(1-f_{S}\right) d x_{L}
$$

Assuming that equilibrium exists at the liquid/solid interface, the average liquid concentration $x_{L}$ at any time can be represented by the solid concentration at the growth front $x_{S}$ in the following equation:

$$
x_{L}=\frac{x_{S}}{k} .
$$

The variable segregation coefficient $k$ is represented by $\mathrm{Ga}$ concentration at the solidus line using linear regression as shown in Figure 2(b) and in the following equation:

$$
k\left(x_{S}\right)=a x_{S}+b \quad(a=0.90, b=0.11) .
$$

By inserting (2) and (3) back into formula (1), the differential equation can be solved as follows:

$$
\begin{aligned}
1- & f_{S} \\
& =\left(\frac{x_{o}}{x_{S}}\right)^{1 /(1-b)}\left(\frac{a x_{S}+b}{a x_{o}+b}\right)\left(\frac{1-b-a x_{S}}{1-b-a x_{o}}\right)^{b /(1-b)} .
\end{aligned}
$$

Equation (4) is the analytical solution of the complete mixing model, where $x_{o}$ is In concentration in the first solidified part of the stripe.

Figure 3 shows a comparison between the model and AES measured data. It can be seen that the complete mixing model (4) agrees with the experimental data. Figure 3(a) shows that the composition profiles are the same for different stripe lengths when the data is plotted against a normalized stripe length, as predicted in (4). Figure 3(b) shows the composition profiles of $25 \mu \mathrm{m}$ long stripes at different cooling rates. All cooling rates result in the same profiles, which suggests that, for short stripe lengths, the profile cannot be modulated by cooling rates. The variation of the data points is likely caused by the Si islands inside the stripes.

Earlier in the paper, in Figure 1(a), we observed small dark regions embedded in the InGaAs stripes. A higher resolution SEM image of one such dark region and AES measurements are shown in Figure 4. Point 1 is measured in the brighter part of the stripe and is seen to be $\operatorname{In}_{x} \mathrm{Ga}_{1-x}$ As. Point 2 is measured in the darker part of the stripe and is seen to be purely Si. This indicates that, for the experimental conditions used in this study, a large amount of Si dissolved from the seed has precipitated into Si grains. The Si signal detected at point 1 is from residual $\mathrm{SiO}_{2}$. 


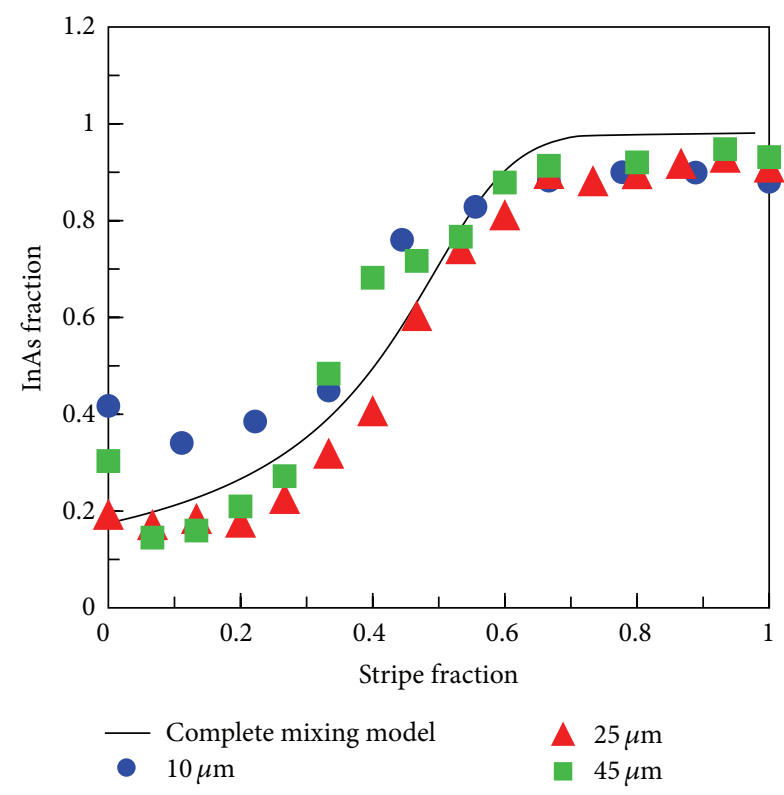

(a)

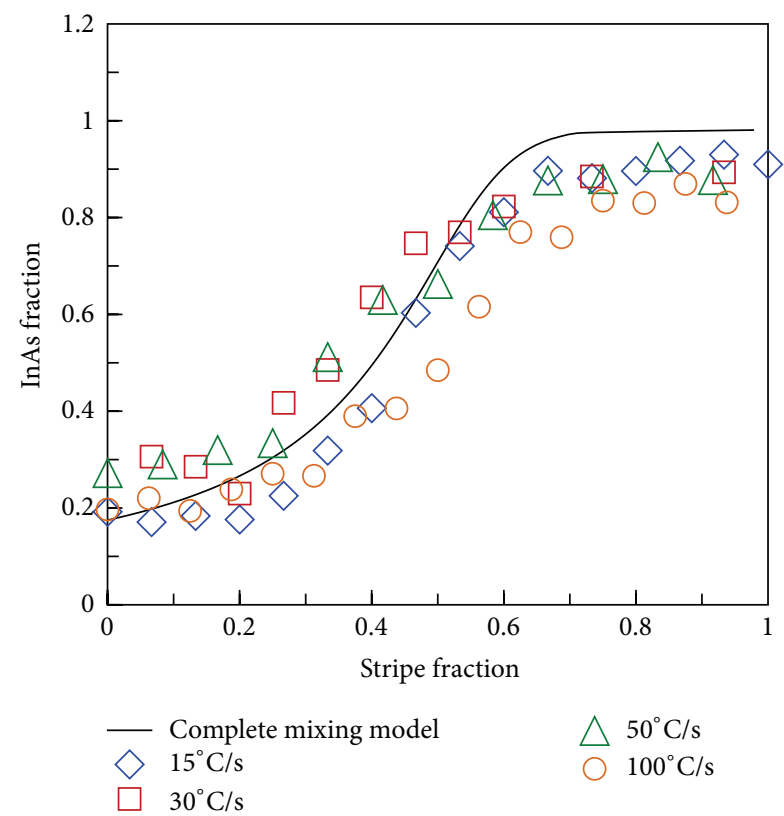

(b)

FIgURE 3: Plots of experimental data and model. (a) shows the InAs composition versus stripe fraction for different stripe length when the initial material is $\operatorname{In}_{0.27} \mathrm{Ga}_{0.2} \mathrm{As}_{0.53}$. (b) shows the InAs composition versus stripe fraction for different cooling rate when the initial material is $\mathrm{In}_{0.27} \mathrm{Ga}_{0.2} \mathrm{As}_{0.53}$.

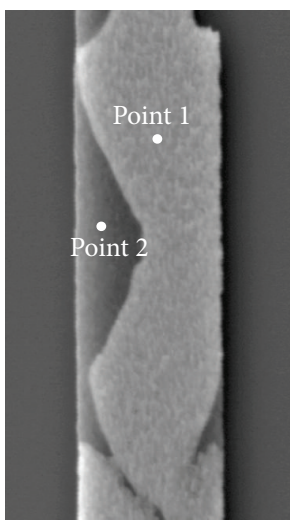

SEM image

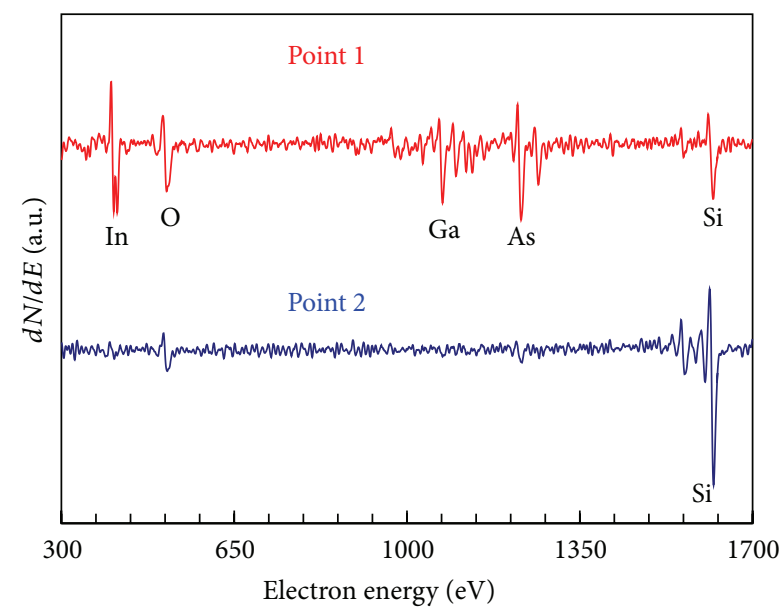

FIGURE 4: SEM image showing that dark region and bright region coexist in InGaAs stripe after RMG. The data shows the compositions of the two color contrast regions.

The reason for the significant Si dissolution can be seen in Figure 5, which is a typical phase diagram for III-V materials and Si. Per the phase diagram, Si and GaAs are completely miscible in the liquid phase. Thus, Si from the substrate will dissolve in the molten GaAs during RMG. During subsequent cooling, due to the low solubility of $\mathrm{Si}$ and GaAs in the solid phase below the eutectic temperature, the dissolved $\mathrm{Si}$ will phase-segregate and crystallize as a second phase. The Si islands can obviously adversely affect the electronic properties of the III-V material and the crystal quality. Our work on eliminating these Si islands from III-V stripes produced by RMG will be described in a subsequent paper.

\section{Conclusion}

In summary, we demonstrate the rapid melt growth of single crystal InGaAs. We studied the segregation effect of In and Ga atoms and developed a complete mixing model that agrees well with the experimental data. Si islands were found in the grown InGaAs materials as a result of eutectic melting and precipitation of $\mathrm{Si}$ which needs to be eliminated if RMG 


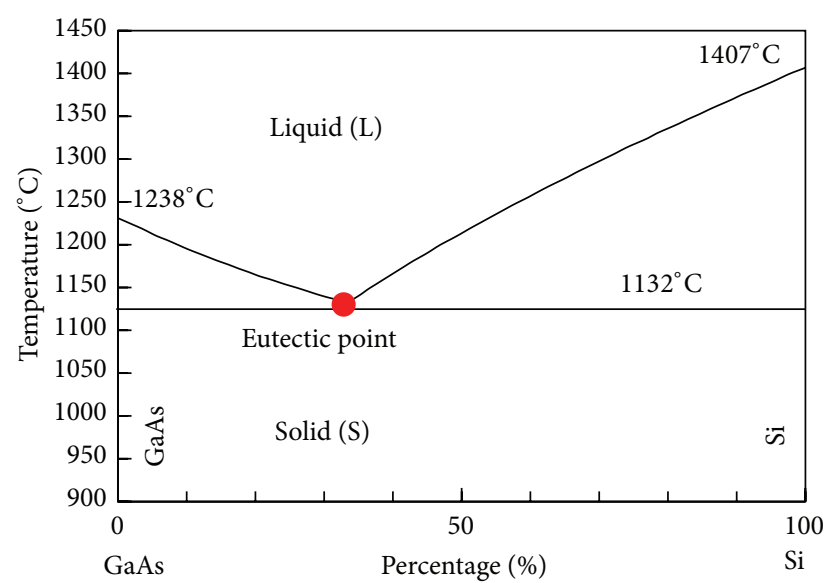

FIgURE 5: Ga-As-Si phase diagram [16] is chosen as a typical phase diagram for III-V-Si system.

III-V materials are to be used to fabricate high performance devices.

\section{Competing Interests}

The authors declare that they have no competing interests.

\section{Acknowledgments}

This work was performed in part in the Stanford Nanofabrication Facility which is supported by the National Science Foundation through the NNIN under Grant ECS-9731293 and in the Stanford Nano Shared Facilities (SNSF) at Stanford University. K. Barton and the Department of Plant Biology, Carnegie Institution, are thanked for SEM access, and J. M. Warren, Stanford University, is thanked for EBSD access. This work was supported by Intel Corporation through the SRC MSR-Intel Liaison Program.

\section{References}

[1] M. Heyns and W. Tsai, "Ultimate scaling of CMOS logic devices with Ge and III-V materials," MRS Bulletin, vol. 34, no. 7, pp. 485-492, 2009.

[2] J. A. del Alamo, "Nanometre-scale electronics with III-V compound semiconductors," Nature, vol. 479, no. 7373, pp. 317-323, 2011.

[3] O. Moutanabbir and U. Gösele, "Heterogeneous integration of compound semiconductors," Annual Review of Materials Research, vol. 40, pp. 469-500, 2010.

[4] K. Nozawa and Y. Horikoshi, "Low threading dislocation density GaAs on $\mathrm{Si}(100)$ with InGaAs/GaAs strained-layer superlattice grown by migration-enhanced epitaxy," Japanese Journal of Applied Physics, vol. 30, no. 4, pp. L668-L671, 1991.

[5] G. Roelkens, J. Brouckaert, D. Taillaert et al., "Integration of InP/InGaAsP photodetectors onto silicon-on-insulator waveguide circuits," Optics Express, vol. 13, no. 25, pp. 10102-10108, 2005.

[6] N. Waldron, G. Wang, N. D. Nguyen et al., "Integration of InGaAs channel n-MOS devices on $200 \mathrm{~mm} \mathrm{Si}$ wafers using the aspect-ratio-trapping technique," ECS Transactions, vol. 45, no. 4, pp. 115-128, 2012.

[7] E. A. Fitzgerald and N. Chand, "Epitaxial necking in GaAs grown on pre-patterned Si substrates," Journal of Electronic Materials, vol. 20, no. 10, pp. 839-853, 1991.

[8] Y. Liu, M. D. Deal, and J. D. Plummer, "High-quality singlecrystal Ge on insulator by liquid-phase epitaxy on Si substrates," Applied Physics Letters, vol. 84, no. 14, pp. 2563-2565, 2004.

[9] Y. Liu, M. D. Deal, and J. D. Plummer, "Rapid melt growth of germanium crystals with self-aligned microcrucibles on $\mathrm{Si}$ substrates," Journal of the Electrochemical Society, vol. 152, no. 8, pp. G688-G693, 2005.

[10] S.-L. Chen, P. B. Griffin, and J. D. Plummer, "Single-crystal gaas and gasb on insulator on bulk Si substrates based on rapid melt growth," IEEE Electron Device Letters, vol. 31, no. 6, pp. 597-599, 2010.

[11] H.-Y. S. Koh, S.-L. Chen, P. B. Griffin, and J. D. Plummer, "High quality single-crystal laterally graded $\mathrm{SiGe}$ on insulator by rapid melt growth," Electrochemical and Solid-State Letters, vol. 13, no. 8, pp. H281-H283, 2010.

[12] X. Bai, C.-Y. Chen, P. B. Griffin, and J. D. Plummer, "Si incorporation from the seed into Ge stripes crystallized using rapid melt growth," Applied Physics Letters, vol. 104, no. 5, Article ID 052104, 2014.

[13] R. Matsumura, Y. Tojo, M. Kurosawa, T. Sadoh, I. Mizushima, and M. Miyao, "Growth-rate-dependent laterally graded SiGe profiles on insulator by cooling-rate controlled rapid-meltinggrowth," Applied Physics Letters, vol. 101, no. 24, Article ID 241904, 2012.

[14] G. B. Stringfellow and P. E. Greene, "Calculation of III-V ternary phase diagrams: In-Ga-As and In-As-Sb," Journal of Physics and Chemistry of Solids, vol. 30, no. 7, pp. 1779-1791, 1969.

[15] E. Scheil, "Bemerkungen zur schichtkristallbildung," Zeitschrift Metallkunde, vol. 34, pp. 70-72, 1942.

[16] V. M. Glazov, "Ga-As-Si phase diagram," in ASM Alloy Phase Diagrams Database, P. Villars, H. Okamoto, and K. Cenzual, Eds., ASM International, Materials Park, Ohio, USA, 2016, http://www.asminternational.org. 

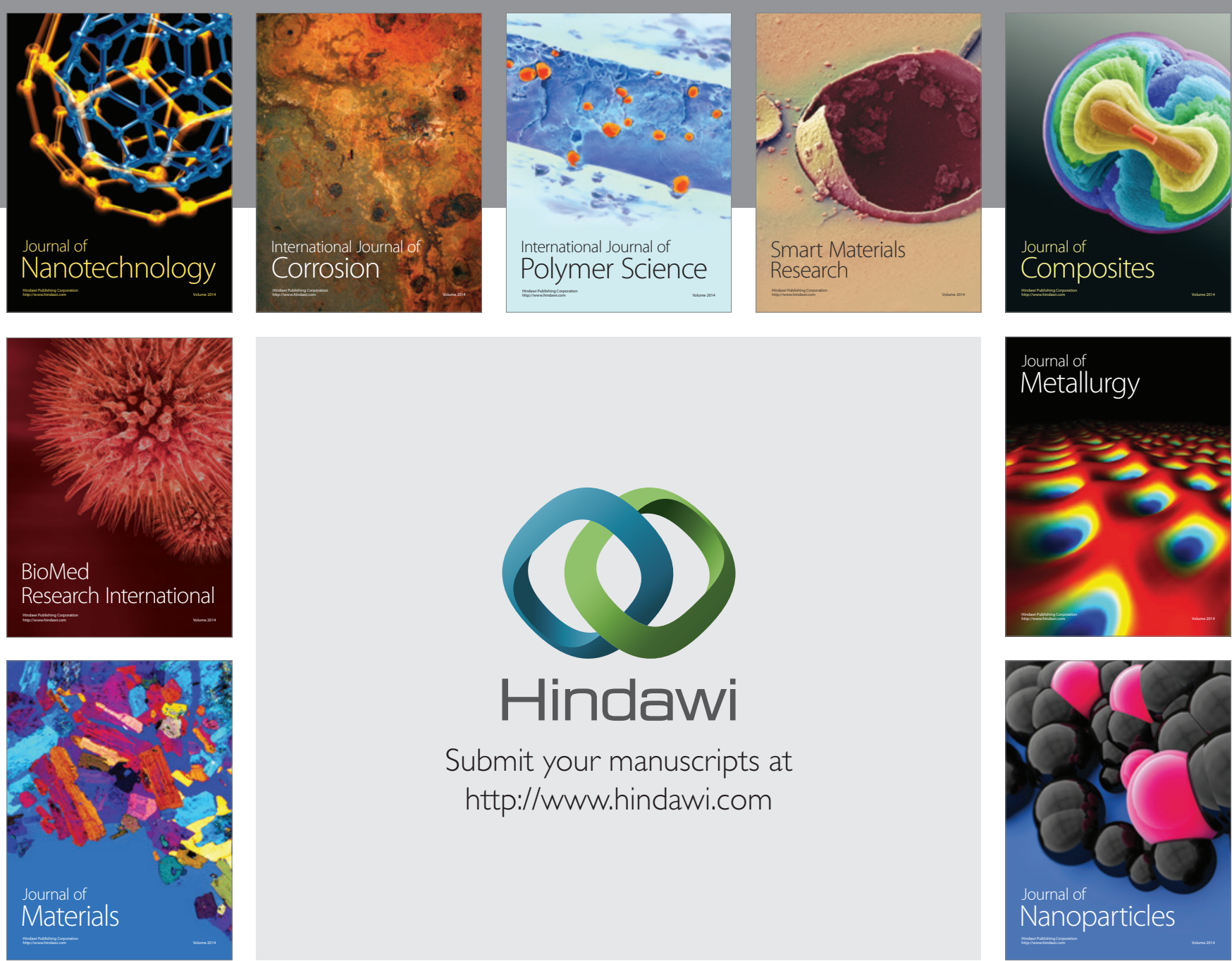

\section{Hindawi}

Submit your manuscripts at

http://www.hindawi.com

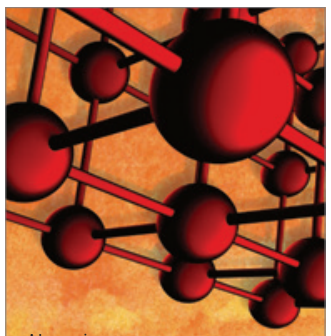

Materials Science and Engineering
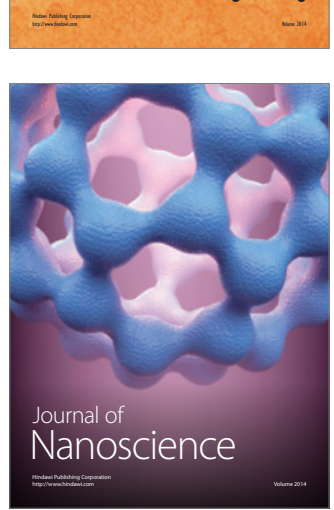

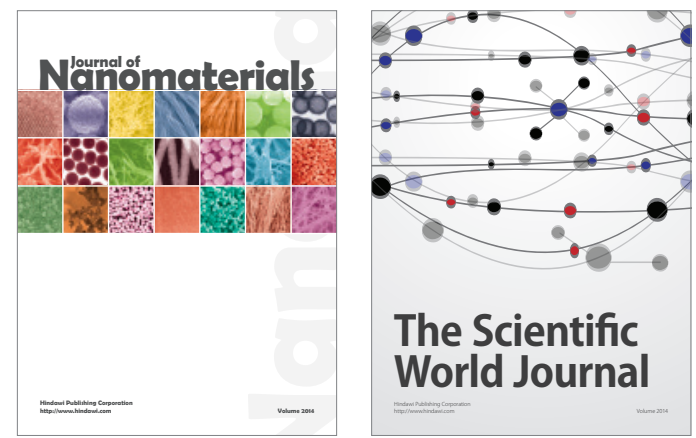

The Scientific World Journal
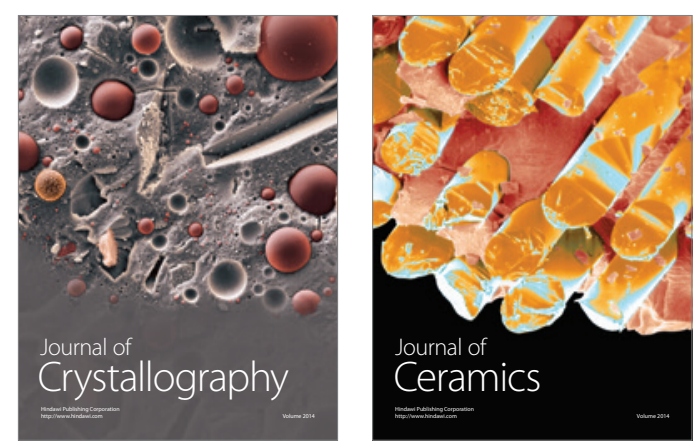
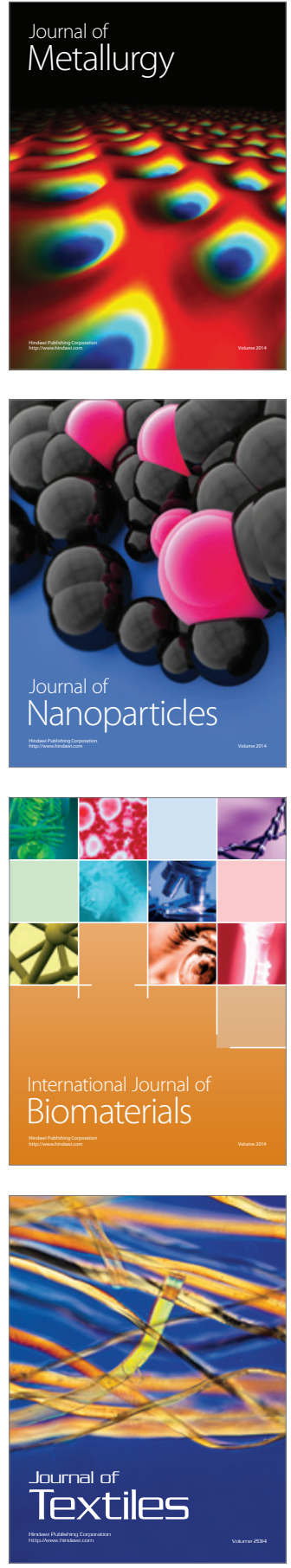